\title{
Immunizations in children with chronic kidney disease
}

\author{
Alicia M. Neu \\ Received: 9 September 2011 /Revised: 4 October 2011 / Accepted: 5 October 2011 /Published online: 3 November 2011 \\ (C) IPNA 2011
}

\begin{abstract}
Children with chronic kidney disease (CKD) are at increased risk for vaccine-preventable diseases. These patients may have a reduced response to and/or reduced duration of antibody after immunization and therefore monitoring of antibody levels or titers is indicated for some vaccines. In addition, pediatric $\mathrm{CKD}$ patients require immunizations not routinely provided to healthy children. Unfortunately, studies in pediatric CKD patients, including those on dialysis and awaiting kidney transplantation, have demonstrated sub-optimal immunization rates. In order to minimize the risk for vaccine-preventable disease in pediatric CKD patients, it is imperative that all who care for these patients remain abreast of the recommended childhood immunization schedule, as well as alterations to this schedule required for children with $\mathrm{CKD}$, including end-stage kidney disease. This article reviews recent changes to the recommended childhood immunization schedule and alterations and additions to this schedule recommended for children with CKD. Where available, data on antibody response to immunizations in children with CKD are presented.
\end{abstract}

Keywords Children · Kidney · Dialysis · Transplantation · Immunization · Vaccination

\section{Introduction}

The care of children with chronic kidney disease (CKD) is very complex and, therefore, delivery of routine well-child

A. M. Neu $(\square)$

Pediatric Nephrology,

The Johns Hopkins University School of Medicine,

200 North Wolfe Street, Room 3065,

Baltimore 21287, USA

e-mail: aneu1@jhmi.edu care, including immunizations, can be delayed or overlooked. In fact, data from the United States Renal Data System (USRDS) reveal that among prevalent end-stage renal disease (ESRD) patients aged $0-19$ years, $32 \%$ or fewer received the seasonal influenza vaccine between 2005 and 2008 , and $13 \%$ or fewer received vaccination against Streptococcal pneumoniae [1]. The importance of maximizing vaccination rates in CKD is highlighted by the inclusion of monitoring of hepatitis B, influenza, and pneumococcal vaccination rates among the indicators for quality assessment and performance improvement programs in the Centers for Medicaid \& Medicare Services' Conditions for Coverage for ESRD facilities. In order to optimally protect children with CKD against vaccinepreventable disease, health care providers who care for these children should be aware of the recent updates to the standard recommendations for immunizations in childhood as well as alterations to this schedule for children with CKD.

\section{Standard recommendations for immunizations in childhood}

The recommended immunization schedule for healthy children in the USA is updated each year by the Center for Disease Control (CDC) Advisory Committee on Immunization Practices (ACIP) and the Committee on Infectious Diseases of the American Academy of Pediatrics (AAP) $[2,3]$. These schedules, with explanatory footnotes, are readily available to health care providers online (http:// www.aap.org/immunization/; http://www.cdc.gov/vaccines/ recs/schedules/child-schedule.htm). In 2011, the AAP and ACIP continue to recommend immunization against diphtheria, tetanus, pertussis, Hemophilus influenzae type b, 
polio, measles, mumps, rubella, varicella-zoster, rotavirus, and hepatitis B for all healthy children. [2, 3] Recent modifications to this schedule include that the quadrivalent human papillomavirus (HPV4) is now recommended for males beginning at age 9 years [2-4]. Females may receive either HPV4 or the bivalent vaccine (HPV2) typically beginning at age 11 years, although it may be given as early as age 9 years [2,3]. In January 2011, the recommendation for a second dose of the quadrivalent meningococcal conjugate vaccine (MCV4) was added to the schedule [5]. It is now recommended that the initial MCV4 dose be given to adolescents, preferably at age 11 or 12 years, with a booster dose at age 16 years. A single dose at age 13 through 18 may be given to patients not vaccinated at age 11 or 12 years [5]. Finally, the 2011 schedule recommends the substitution of the 13-valent conjugated pneumococcal vaccine (PCV13) for the previously recommended 7-valent conjugated pneumococcal vaccine (PCV7) $[6,7]$.

In addition to the annually updated recommendations for healthy children, health care providers caring for children with CKD should be aware of a guideline for vaccinating patients on dialysis and with CKD, which was published by the CDC in 2006 and which is available on the CDC website. (http://www.cdc.gov/vaccines/pubs/downloads/ b_dialysis_guide-508.pdf). [8] In general, children with CKD should receive all of the recommended childhood immunizations according to the standard schedule, whenever possible. However, the live-viral vaccines (rotavirus vaccine, varicella vaccine, measles, mumps, and rubella vaccine, and the live-attenuated influenza vaccine) should be avoided in patients who require treatment with immunosuppressive medication, including those with status postkidney transplant. In the following sections, available data on the antibody response to the various vaccines in pediatric CKD patients will be provided, and any modification of the standard schedule that may be required for children with CKD will be discussed.

\section{Immunizations in children with CKD}

Diphtheria, tetanus and pertussis vaccine

Immunization against diphtheria, tetanus and pertussis has long been recommended for healthy infants [2, 3]. The vaccination series includes a total of five doses, the first four of which are given at ages 2, 4, 6, and 15-18 months, and the fifth at age 4-6 years [2,3]. There are several studies that suggest that children and young adults with CKD, including those on dialysis, have sub-optimal seroconversion rates following immunization with diphtheria and tetanus toxoids and whole-cell pertussis (DTP) vaccines [9-11]. However, a multi-center studied performed in infants vaccinated with DTP while on dialysis revealed protective antibody titers to both diphtheria and tetanus toxoids in seven of the eight patients $(88 \%)$ [12]. Since 1997, the ACIP has recommended that vaccines containing the less reactogenic acellular pertussis antigens (DTaP) replace DTP [13]. In 2005, a tetanus toxoid, reduced diphtheria, and acellular pertussis vaccine (Tdap) was licensed, and the use of this vaccine has been recommended to replace the tetanus diphtheria booster (Td) dose in adolescents since 2006. [14] Although data on the response to the acellular pertussis vaccines in children with CKD are lacking, it is currently recommended that infants with CKD receive DTaP according to the standard childhood schedule. Given the data on older children on dialysis, efforts should be made to ensure that immunization with Tdap and subsequent booster immunizations are provided in a timely fashion.

Hemophilus influenzae type b (Hib) conjugate vaccine

Immunization against Hemophilus influenzae type b (Hib) is recommended for all infants, with a four-dose series beginning at age 2 months and ending between 12 and 15 months [2,3]. A multi-center study in infants vaccinated with Hib conjugate vaccine while on dialysis found that nine of the ten $(90 \%)$ patients had protective antibody levels after vaccination and that antibody levels remained protective for as long as 22 months post-vaccination [15]. A second study found protective antibody levels 2 months after the third dose of Hib conjugate vaccine in all 42 pediatric dialysis patients studied [16]. Thus, children with CKD should receive this vaccine according to the standard schedule.

\section{Hepatitis B vaccine}

Vaccination against hepatitis B is currently recommended for all infants, with the first dose given to newborns before hospital discharge $[2,3]$. The second dose is to be given at age 1 or 2 months, with a final dose between 6 and 18 months of age $[2,3]$. Because dialysis, and hemodialysis in particular, is associated with a significantly increased risk for hepatitis B infection, every effort should be taken to minimize that risk with vaccination $[8,17]$. Unfortunately, a suboptimal response to hepatitis $\mathrm{B}$ vaccine and a more rapid decline in anti-hepatitis $\mathrm{B}$ antibody levels after immunization have been well-documented among adult dialysis patients and, therefore, the ACIP recommends that adult patients on dialysis receive an augmented dose of $40 \mu \mathrm{g}$ of either Recombivax HB (Merck \& Co. Whitehouse Station, NJ) or Engerix-B (GlaxoSmithKline, London, UK) $[8,17]$. Studies in adult CKD patients suggest that the adjuvant 3-O-desacyl-40-monophosphoryl lipid A may 
induce higher and more durable antibody levels, and such an adjuvanted hepatitis B vaccine has been licensed in Europe for use in adult CKD patients [18, 19]. However, this vaccine is not currently in use in the USA, and there are no studies of the vaccine in children with CKD [18, 19]. Data regarding the response to standard recombinant hepatitis vaccines in pediatric CKD are limited. One study vaccinated 62 children on dialysis or status post-kidney transplant with a three-dose series of $5 \mu \mathrm{g}$ (age $<10$ years) or $10 \mu \mathrm{g}$ (age $>10$ years) hepatitis $\mathrm{B}$ surface antigen (HbsAg) [16]. Antibody levels obtained 2 months after the final immunization were protective in $60 / 62$ (97\%) patients [16]. In a multi-center study performed by the Southwest Pediatric Nephrology Study Group, 78 pediatric patients with CKD pre-dialysis, on dialysis, or with status postkidney transplant were given three immunizations of $20 \mu \mathrm{g}$ HbsAg after which $91 \%$ of patients had a protective titer of $\geq 10 \mathrm{mIU} / \mathrm{ml}$. [20] A lower percentage of patients immunized post-transplant had protective antibody levels in comparison with those with CKD pre-dialysis or on dialysis (66.7 vs. $96.4 \%$ ) [20]. In addition, mean geometric mean antibody titers after three immunizations tended to be higher in patients with CKD pre-dialysis than in patients on dialysis or post-transplant, leading the authors to suggest that at least two immunizations be given prior to the point at which dialysis or transplant are necessary, whenever possible [20].

Although these data suggest that pediatric CKD patients may benefit from an augmented dose of hepatitis B vaccine, at present the ACIP recommends that $\mathrm{CKD}$ and dialysis patients younger than 20 years of age receive hepatitis $\mathrm{B}$ immunization according to the standard schedule, with the caveat that "higher doses might be more immunogenic" in pediatric hemodialysis patients $[8,11,21]$. Post-vaccination testing is recommended 1-2 months after the primary series is completed, with up to three additional doses if protective antibody levels $(>10 \mathrm{mIU} / \mathrm{ml})$ are not achieved [8, 11]. Antibody levels should then be measured annually and booster doses provided to patients whose antibody levels fall below protective [8].

\section{Inactivated polio virus vaccine}

Since 1999, AAP and ACIP recommendations have specified that only inactivated polio virus (IPV) vaccine, rather than the live-attenuated oral polio vaccine, be used for routine immunization in all children beginning at age 2 months, with a second dose at 4 months, a third dose between 6 and 18 months, and the final dose between 4 and 6 years of age $[2,3,22]$. Although IPV vaccine should routinely be delivered to infants with $\mathrm{CKD}$, there are no studies documenting the response to this vaccine in this patient population. A study performed in older children on dialysis measured antibody levels after vaccination with IPV vaccine and found that $42 / 49(86 \%)$ patients either had protective antibody levels to all three serotypes prior to vaccination or had at least a fourfold increase in antibody levels following immunization [23]. Because this vaccine contains only inactivated virus, it may be given to CKD patients who are also on immunosuppressive medications as well as their household contacts.

Measles, mumps, rubella vaccine

Vaccination against measles, mumps and rubella (MMR) is recommended for all children between the ages of 12 and 15 months, with a second dose typically between 4 and 6 years of age $[2,3]$. MMR vaccine is a live, attenuated viral vaccine and as such should be avoided in children with CKD if they are receiving immunosuppressive therapy, including corticosteroids at a dose greater than $2 \mathrm{mg} / \mathrm{kg}$ body weight or $20 \mathrm{mg}$ total daily or on alternate days for more than 14 days [24]. Once corticosteroids are discontinued, it is generally recommended that MMR vaccination be delayed for at least 1 month. [24] In addition, this live-viral vaccine is contraindicated in the immunosuppressed patient status post-kidney transplant [24].

There have been several studies evaluating the response to MMR in pediatric CKD patients, including a study of ten dialysis patients vaccinated with MMR between 15 and 33 months of age following which only $70 \%$ developed protective titers to measles, $50 \%$ to mumps, $80 \%$ to rubella, and only three of then (30\%) to all three viruses [25]. An additional study vaccinated nine infants, six of whom were on dialysis, at a mean age of 11.6 months [26]. Eight of these patients were subsequently transplanted at a mean age of 16 months, and at the time of transplantation, $89 \%$ had protective titers to measles, $88 \%$ to mumps, $100 \%$ to rubella, and $88 \%$ to all three viruses [26]. In a third study, positive antibody titers were found in $100 \%$ of 62 pediatric dialysis patients 2 months after immunization with MMR [16]. Although these data suggest that many pediatric patients on dialysis may respond well to MMR vaccine, because immunization post-transplant is contraindicated, antibody titers should be measured prior to proceeding to transplant, and repeat vaccination should be given to patients with negative titers [24].

Varicella zoster virus vaccine

Immunization with varicella zoster virus (VZV) vaccine was first implemented in the USA in 1995, with a single dose to be administered at 12-15 months of age [27]. However, outbreaks of varicella continued to occur, even in settings with high vaccination coverage, and thus a second 
dose of varicella vaccine, to be given at 4-6 years of age, was added to the routine childhood vaccination program in 2006 [28].

VZV vaccine is another live, attenuated viral vaccine and is therefore contraindicated in CKD patients on immunosuppressive medication, including those status post-kidney transplantation [27]. There have been several studies evaluating the immunogenicity of this vaccine in children with $\mathrm{CKD}$, including studies using the previously recommended single immunization with VZV vaccine which demonstrated seroconversion rates of $85-88 \%$, compared to a rate of $99 \%$ in healthy children $[29,30]$. Two multi-center, prospective studies evaluated antibody levels in children with CKD after a two-dose regimen of VZV vaccine [31, 32]. Both studies revealed that nearly all patients seroconverted after the second dose of vaccine, with a $98 \%$ seroconversion rate in one study and $100 \%$ in the other [31, 32]. Few infants were included in these studies, however, and thus seroconversion rates in infants and toddlers with CKD after either a one- or two-dose regimen are not known. Given the significant risk for morbidity and mortality from varicella infection status post-kidney transplantation, it is reasonable to consider measuring antibody levels prior to kidney transplantation in any patient without a history of wild-type infection and to provide supplemental vaccination if positive antibody titers are not demonstrated.

\section{Pneumococcal vaccine}

Immunization against Streptococcus pneumonia using the 23-valent polysaccharide pneumococcal vaccine (PPSV23) has been recommended for children with nephrotic syndrome since 1989 and for children with CKD since 1997, as these patients are considered to be at high risk for the development of invasive pneumococcal infection [33, 34]. This vaccine is poorly immunogenic in infants, however, and therefore its use is limited to children 24 months and older [33, 34]. In 2000, a conjugated pneumococcal vaccine, PCV7, which is highly immunogenic in infants, was licensed for use in the USA, and subsequently routine immunization with PCV7 at ages 2, 4, 6, and 12-15 months was recommended for all children, including those with CKD [35]. Studies of the PCV7 vaccine have revealed adequate antibody response in children with $\mathrm{CKD}$, those on dialysis, and solid organ transplant recipients [36, 37].

As mentioned previously, in 2011 the AAP and ACIP recommended that a newly licensed PCV13 replace the PCV7 in both healthy children and those with kidney disease, with the aim of expanding serotype coverage [2, 3, $6,7]$. The number and timing of doses for PCV13 in children under 24 months is based on the number of previous immunizations with PCV7 [6, 7]. For children with kidney disease aged 24-71 months who received at least three doses of either PCV7 or PCV13 before age 24 months, a single dose of PCV13 is recommended at least 8 weeks after the most recent dose [6, 7]. A two-dose series of PCV13 is to be given to children with kidney disease aged 24-71 months and to children either previously unvaccinated or with fewer than three previous doses of either conjugated vaccine (PCV7 or PCV13) [3, 4].

It continues to be recommended that children with $\mathrm{CKD}$ and nephrotic syndrome over the age of 2 years receive supplemental immunization with PPSV23 [6, 7]. The vaccine should be given at least 8 weeks after the final dose of PCV13 [6, 7]. Revaccination with PPSV23 had previously been recommended after 3 years in children younger than 10 years of age at the time of first PPSV23 vaccination, and 5 years after the initial immunization in older children, but the new recommendations suggest a second dose of PPSV23 after 5 years for all children with CKD $[6,7]$.

For children ages 6 through 18 years of age with CKD, the new recommendations suggest that a single dose of PCV13 should be given regardless of their previous history of PCV7 or PPSV23 $[6,7]$. Children with CKD who have not previously received PPSV23 should receive vaccination with PPSV23 at least 8 weeks after PCV13, with a repeat dose of PPSV23 after 5 years [6, 7]. The recommended schedules according to age and history of pneumococcal vaccination are provided in detail on the AAP and CDC websites (http://www.aap.org; http://www. cdc.gov/vaccines/recs/acip).

Hepatitis A vaccine, meningococcal vaccine, human papillomavirus vaccine, and rotavirus vaccine

There are currently no data available on the response to the hepatitis A, meningococcal, human papillomavirus (HPV) or rotavirus vaccines in children with $\mathrm{CKD}$, although prospective studies of the response to HPV and the tetravalent conjugated meningococcal vaccine in children with $\mathrm{CKD}$, including dialysis and transplant patients, are currently underway (A. Neu, personal communication). Children with CKD may receive these vaccines as recommended for healthy children with the caveat that the live-attenuated rotavirus vaccine be avoided in children on immunosuppressive therapy.

Influenza vaccine

Influenza vaccination was previously recommended only for high-risk populations, but annual vaccination against influenza is now recommended for all children over the age of 6 months [38]. High-risk populations, including children with CKD, continue to be a priority for immunization [39]. 
The composition of the influenza vaccine changes each year based on the strains of viruses likely to circulate in the upcoming year and, therefore, this vaccine must be given annually, typically in the fall [39]. Children under the age of 9 years who are receiving the influenza vaccine for the first time should receive two doses, given at least 1 month apart [39]. The influenza vaccine is available either as an inactivated vaccine or a live, attenuated vaccine (LAIV). However, the CDC currently recommends that persons on dialysis and those with the status post-kidney transplant do not receive LAIV. Therefore, only the inactivated vaccine should be given to children on dialysis and status postkidney transplant [40].

There have been several studies evaluating vaccine response in pediatric CKD patients. The majority of these studies have focused on pediatric kidney transplant patients, and although one study revealed a lower seroconversion rate than in healthy siblings, two other studies have demonstrated seroconversion rates that are similar between transplant patients and controls [41-43]. One of these studies also evaluated vaccine response in pediatric dialysis patients and revealed equivalent seroconversion rates between the study group and controls [42]. Although these studies suggest that influenza vaccine produces a reasonable response in pediatric CKD patients, because of the significant risk for morbidity and mortality from influenza infection in these patients, household contacts should receive vaccination in an effort to decrease the risk for exposure to wild-type influenza [39].

\section{Summary}

Children with CKD are at increased risk for vaccinepreventable diseases. Unfortunately, CKD may also reduce the immunologic response to some vaccines. It should also be recognized that vaccine response does not necessarily confer immunity, and there are reports of Hemophilus influenzae infections and pneumococcal peritonitis despite adequate antibody response to vaccines against the causal pathogens [16]. Nevertheless, immunization remains one of the cornerstones of prevention. Pediatric CKD patients should receive all of the vaccines currently recommended for healthy children according to the standard schedule, with the exception of the avoidance of the live-viral vaccines (rotavirus vaccine, MMR, VZV, LAIV) in patients receiving immunosuppressive medication and the avoidance of the LAIV in dialysis patients [24, 27, 40]. Because MMR and VZV vaccines are contraindicated posttransplant, every effort to provide immunization prior to the introduction of immunosuppressive medication posttransplantation should be made. In addition, protection against MMR and VZV should be documented prior to transplantation and revaccination considered in those patients without protective antibody titers. The timing of resumption of non-live vaccination series post-transplantation is variable among centers, but many delay immunizations for 6 months post-transplant, as recommended by the American Society of Transplantation (AST). [44]

Children with CKD are considered to be at high risk for infection with hepatitis $\mathrm{B}$, and data suggest that some of these patients may benefit from an augmented dose of this vaccine $[8,11,20]$. At the very least, antibody levels should be monitored and additional doses provided if an antibody level $>10 \mathrm{mIU} / \mathrm{ml}$ is not achieved after completion of the three shot series [11]. In addition, antibody levels should be monitored yearly, with a booster dose if levels fall below $10 \mathrm{mIU} / \mathrm{ml}$ [11]. Pediatric CKD patients should receive supplemental immunization with the 13-valent conjugated and 23-valent polysaccharide pneumococcal vaccines $[6,7]$. The number and timing of these supplemental vaccines depends on the patient's age and vaccination history. In addition, revaccination with PPSV23 is indicated 5 years after the initial vaccination. Finally, influenza vaccines should be given annually to all pediatric CKD patients older than 6 months and to their household contacts.

Acknowledgments Dr. Neu has received or is receiving grant support from Sanofi-Pasteur and Merck \& Co., the manufacturers of some of the vaccines discussed in this review.

\section{Questions (answers are provided following the reference list)}

1.) Children with CKD require alteration or augmentation to the standard immunization schedule for healthy children for which of the following vaccines?

a. Diphtheria, tetanus and acellular pertussis accine

b. Hemophilus influenza type $b$ vaccine

c. Human papillomavirus vaccine

d. 13-Valent pneumococcal vaccine

e. Conjugated meningococcal vaccine

2.) Which of the following vaccines is contraindicated in children with CKD treated with immunosuppressive medications, including those status post-kidney transplant?

a. Diphtheria, tetanus and acellular pertussis

b. Human papillomavirus

c. Varicella zoster virus vaccine

d. Inactivated poliovirus vaccine

e. Conjugated meningococcal vaccine

3.) Which of the following statements regarding influenza vaccination is TRUE?

a. Vaccination should be provided to children with CKD every 5 years 
b. All children with CKD may receive the liveattenuated influenza virus vaccine

c. All household contacts of children with CKD should receive annual influenza vaccine

d. Vaccination should be avoided in children with CKD on immunosuppressive medication

e. Vaccination should only be provided to CKD patients over the age of 24 months

4.) Annual measurement of antibody to which of the following is recommended in children on dialysis?
a. Hepatitis B
b. Hepatitis A
c. Varicella zoster virus
d. Measles
e. Rubella

5.) Which of the following statements regarding the PCV13 in pediatric CKD patients is TRUE?

a. It should be given only to children younger than 24 months of age

b. It may be given to children with previous vaccination with the PPSV23

c. It is not required in children with previous vaccination with the PCV7

d. It should be repeated 5 years after the initial immunization

\section{References}

1. U.S. Renal Data System (2010) USRDS 2010 annual data report: atlas of chronic kidney disease and end-stage denal disease in the United States. National Institute of Diabetes and Digestive and Kidney Diseases, National Institutes of Health, Bethesda

2. Committee of Infectious Diseases (2011) Recommended childhood and adolescent immunization schedules United States, 2011. Pediatrics 127:387-388

3. Centers for Disease Control and Prevention (2011) Recommended immunization schedules for persons aged 0 through 18 yearsUnited States, 2011. MMWR Morb Mortal Wkly Rep 60(5):1-4

4. ACIP Provisional Recommendations for HPV vaccine. Available at: http://www.cdc.gov/vaccines/vpd-vac/hpv/default.htm

5. Centers for Disease Control and Prevention (2011) Updated recommendations for use of meningococcal conjugate vaccinesAdvisory Committee on Immunization Practices (ACIP), 2010. MMWR Morb Mortal Wkly Rep 60(3):72-76

6. Centers for Disease Control and Prevention (2010) Prevention of pneumococcal disease among infants and children-use of 13valent pneumococcal conjugate vaccine and 23 -valent pneumococcal polysaccharide vaccine. Recommendations of the Advisory Committee on Immunization Practices (ACIP). MMWR Morb Mortal Wkly Rep 59(11):1-18

7. American Academy of Pediatrics (2010) Policy StatementRecommendations for the prevention of Streptococcus pneumoniae infections in infants and children: use of 13-valent pneumococcal conjugate vaccine (PCV13) and pneumococcal polysaccharide vaccine (PPSV23). Pediatrics 126:186-190
8. Centers for Disease Control and Prevention. Guidelines for vaccinating kidney dialysis patients and patients with chronic kidney disease: Summarized from recommendations of the Advisory Committee on Immunization Practices. Available at: http://www. cdc.gov/vaccines/pubs/downloads/b_dialysis_guide-508.pdf.

9. Ghio L, Pedrazzi C, Assael BM, Panuccio A, Foti M, Edefonti A (1997) Immunity to diphtheria and tetanus in a young population on a dialysis regimen or with a renal transplant. J Pediatr 130:987-989

10. Girndt M, Pietsch M, Kohler H (1995) Tetanus immunization and its association to hepatitis B vaccination in patients with chronic renal failure. Am J Kidney Dis 26:454-460

11. Rangel MC, Coronado VG, Euler GL, Strikas RA (2000) Vaccine recommendations for patients on chronic dialysis. The Advisory Committee on Immunization Practices and the American Academy of pediatrics. Semin Dial 13:101-107

12. Neu AM, Warady BA, Furth SL, Lederman HM, Fivush BA (1997) Antibody levels to diphtheria, tetanus, and rubella in infants vaccinated while on PD: A study of the Pediatric Peritoneal Dialysis Study Consortium. Adv Perit Dial 13:297-299

13. Centers for Disease Control and Prevention (1997) Pertussis vaccination: use of acellular pertussis vaccines among infants and young children. Recommendations of the Advisory Committee on Immunization Practices (ACIP). MMWR Morb Mortal Wkly Rep46(7):1-25

14. Centers for Disease Control and Prevention (2006) Preventing tetanus, diphtheria, and pertussis among adolescents: use of tetanus toxoid, reduced diphtheria toxoid and acellular pertussis vaccines. Recommendations of the Advisory Committee on Immunization Practices (ACIP). MMWR Morb Mortal Wkly Rep 55(3):1-34

15. Neu AM, Lederman HM, Warady BA, Furth SL, Fivush BA (1998) Haemophilus influenza type b immunization in infants on peritoneal dialysis. Pediatr Nephrol 10:84-85

16. Laube GF, Berger C, Goetschel P, Leumann E, Neuhaus TJ (2002) Immunization in children with chronic renal failure. Pediatr Nephrol 17:638-642

17. Centers for Disease Control and Prevention (2001) Recommendations for preventing transmission of infections among chronic hemodialysis patients. MMWR Morb Mortal Wkly Rep 50(5):1-46

18. Tong NK, Beran J, Kee SA, Miguel JL, Sánchez C, Bayas JM, Vilella A, de Juanes JR, Arrazola P, Calbo-Torrecillas F, de Novales EL, Hamtiaux V, Lievens M, Stoffel M (2005) Immunogenicity and safety of an adjuvanted hepatitis B vaccine in pre-hemodialysis and hemodialysis patients. Kidney Int 68:2298-2303

19. Kong NC, Beran J, Kee SA, Miguel JL, Sánchez C, Bayas JM, Vilella A, Calbo-Torrecillas F, López de Novales E, Srinivasa K, Stoffel M, Hoet B (2008) A new adjuvant improves the immune response to hepatitis B vaccine in hemodialysis patients. Kidney Int 73:856-862

20. Watkins SL, Alexander SR, Brewer ED, Hesley TM, West DJ, Chan IS, Mendelman P, Bailey SM, Burns JL, Hogg RJ, Southwest Pediatric Nephrology Study Group (2002) Response to recombinant hepatitis $\mathrm{B}$ vaccine in children and adolescents with chronic renal failure. Am J Kidney Dis 40:365-372

21. Centers for Disease Control and Prevention (2005) A comprehensive immunization strategy to eliminate transmission of Hepatitis B virus infection in the United States: Recommendations of the Advisory Committee on Immunization Practices (ACIP) Part 1: Immunization of infants, children, and adolescents. MMWR Morb Mortal Wkly Rep 54(16):1-33

22. American Academy of Pediatrics Committee on Infectious Diseases (1999) Prevention of poliomyelitis. Recommendations for use of only inactivated polio vaccine for routine immunization. Pediatrics 104:1404-1406 
23. Sipilä R, Hortling L, Hovi T (1990) Good seroresponse to enhanced-potency inactivated poliovirus vaccine in patients on chronic dialysis. Nephrol Dial Transplant 5:352-355

24. Centers for Disease Control and Prevention (1998) Measles, mumps, and rubella vaccine use and strategies for elimination of measles, rubella and congenital rubella syndrome and control of mumps: Recommendations of the Advisory Committee on Immunization Practices (ACIP). MMWR Morb Mortal Wkly Rep 47(08):1-58

25. Schulman SL, Deforest A, Kaiser BA, Polinsky MS, Baluarte HJ (1992) Response to measles-mumps-rubella vaccine in children on dialysis. Pediatr Nephrol 6:187-189

26. Flynn JT, Frisch K, Kershaw DB, Sedman AB, Bunchman TE (1999) Response to early measles-mumps-rubella vaccination in infants with chronic renal failure and/or receiving peritoneal dialysis. Adv Perit Dial 15:269-272

27. Centers for Disease Control and Prevention (1996) Prevention of varicella: recommendations of the Advisory Committee on Immunization Practices (ACIP). MMWR Morb Mortal Wkly Rep 45:1-36

28. Centers for Disease Control and Prevention (2007) Prevention of varicella: recommendations of the Advisory Committee on Immunization Practices (ACIP). MMWR Morb Mortal Wkly Rep 56(4):1-40

29. Broyer M, Boudailliez B (1985) Varicella vaccine in children with chronic renal insufficiency. Postgrad Med J 61(4):103-106

30. Zamora I, Simon JM, Da Silva ME, Piqueras AI (1994) Attenuated varicella virus vaccine in children with renal transplants. Pediatr Nephrol 8:190-192

31. Furth SL, Hogg RJ, Tarver J, Moulton LH, Chan C, Fivush BA, Southwest Pediatric Nephrology Study Group (2003) Varicella vaccination in children with chronic renal failure: A report of the Southwest Pediatric Nephrology Study Group. Pediatric Nephrol $18: 33-38$

32. Webb NJ, Fitzpatrick MM, Hughes DA, Brocklebank TJ, Judd BA, Lewis MA, Postlethwaite RJ, Smith PA, Corbitt G (2000) Immunisation against varicella in end stage and pre-end stage renal failure. Varicella vaccination in children with chronic renal failure: A report of the Southwest Pediatric Nephrology Study Group. Arch Dis Child 82:141-143

33. Centers for Disease Control and Prevention (1989) Recommendations of the Immunization Practices Advisory Committee. Pneumococcal polysaccharide vaccine. MMWR Morb Mortal Wkly Rep 38(5):64-68, 73-76

34. Centers for Disease Control and Prevention (1997) Prevention of pneumococcal disease. Recommendations of the Advisory Committee on Immunization Practices (ACIP). MMWR Morb Mortal Wkly Rep 46(8):1-24

35. American Academy of Pediatrics Committee on Infectious Diseases (2000) Recommendations for the prevention of pneumococcal infections, including the use of pneumococcal conjugate vaccine (Prevnar), pneumococcal polysaccharide vaccine, and antibiotic prophylaxis. Pediatrics 106:362-366

36. Vieira S, Baldacci ER, Carneiro-Sampaio M, Filho D, Koch VY (2009) Evaluation of antibody response to the heptavalent pneumococcal conjugate vaccine in pediatric chronic kidney disease. Pediatr Nephrol 24:83-89

37. Barton M, Wasfy S, Dipchand AI, Hébert D, Ng V, Solomon M, Fecteau A, Stephen D, Allen U (2009) Seven-valent pneumococcal conjugate vaccine in pediatric solid organ transplant recipients. Pediatr Infect Dis J 28:688-692

38. Centers for Disease Control and Prevention (2008) Prevention and control of influenza. Recommendations of the Advisory Committee on Immunization Practices (ACIP), 2008. MMWR MMWR Morb Mortal Wkly Rep 57(07):1-60

39. Centers for Disease Control and Prevention (2002) Prevention and control of influenza. Recommendations of the Advisory Committee on Immunization Practices (ACIP). MMWR MMWR Morb Mortal Wkly Rep 51(03):1-32

40. Centers for Disease Control and Prevention (2005) Prevention and control of influenza. Recommendations of the Advisory Committee on Immunizations Practices (ACIP). MMWR MMWR Morb Mortal Wkly Rep 54(8):1-40

41. Edvardsson VO, Flynn JT, Deforest A, Kaiser BA, Schulman SL, Bradley A, Palmer J, Polinsky MS, Baluarte HJ (1996) Effective immunization against influenza in pediatric renal transplant recipients. Clin Transplant 10:556-560

42. Furth SL, Neu AM, McColley SA, Case B, Steinhoff M, Fivush B (1995) Immune response to influenza vaccination in children with renal disease. Pediatr Nephrol 9:566-568

43. Mauch TJ, Crouch NA, Freese DK, Braunlin EA, Dunn DL, Kashtan CE (1995) Antibody response of pediatric solid organ transplant recipients to immunization against influenza virus. $\mathrm{J}$ Pediatr 127:957-960

44. (2004) Guidelines for vaccination of solid organ transplantation. Am J Transplant 4 [Suppl 10]:160-163

\section{Answers:}

1. d. 13-valent Pneumococcal Vaccine

2. c. Varicella zoster virus vaccine

3. c. All household contacts of children with CKD should receive annual influenza vaccine

4. a. Hepatitis B

5. b. It may be given to children with previous vaccination with the PPSV23 